\title{
A Lyman- $\alpha$ blob in the GOODS South field: evidence for cold accretion onto a dark matter halo ${ }^{\star}$
}

\author{
K. K. Nilsson ${ }^{1,2}$, J. P. U. Fynbo ${ }^{2}$, P. Møller ${ }^{1}$, J. Sommer-Larsen² ${ }^{2}$ and C. Ledoux ${ }^{3}$ \\ 1 European Southern Observatory, Karl-Schwarzschild-Straße 2, 85748 Garching bei München, Germany \\ e-mail: knilsson@eso.org \\ 2 DARK Cosmology Centre, Niels Bohr Institute, University of Copenhagen, Juliane Maries Vej 30, 2100 Copenhagen $\varnothing$, Denmark \\ 3 European Southern Observatory, Alonso de Córdova 3107, Casilla 19001, Vitacura, Santiago 19, Chile
}

Received 14 December 2005 / Accepted 29 April 2006

ABSTRACT

\begin{abstract}
We report on the discovery of a $z=3.16$ Lyman- $\alpha$ emitting blob in the Great Observatories Origins Deep Survey (GOODS) South field. The discovery was made with the VLT, through narrow-band imaging. The blob has a total Ly $\alpha$ luminosity of $\sim 10^{43} \mathrm{erg} \mathrm{s}^{-1}$ and a diameter larger than $60 \mathrm{kpc}$. The available multi-wavelength data in the GOODS field consists of 13 bands from X-rays (Chandra) to infrared (Spitzer). Unlike other known Ly $\alpha$ blobs, this blob shows no obvious continuum counter-parts in any of the broad-bands. In particular, no optical counter-parts are found in deep HST/ACS imaging. For previously published blobs, AGN (Active Galactic Nuclei) or "superwind" models have been found to provide the best match to the data. We here argue that the most probable origin of the extended Ly $\alpha$ emission from this blob is cold accretion onto a dark matter halo.
\end{abstract}

Key words. cosmology: observations - galaxies: high redshift - galaxies: halos

\section{Introduction}

Narrow-band surveys for Lyman- $\alpha(\operatorname{Ly} \alpha)$ emitting galaxies at high redshift have recently revealed a number of luminous (up to $5 \times 10^{43} \mathrm{erg} \mathrm{s}^{-1}$ ), very extended (from a few times ten $\mathrm{kpc}$ to more than $150 \mathrm{kpc}$ ) Ly $\alpha$-emitting objects, so-called Ly $\alpha$ "blobs" (Fynbo et al. 1999; Keel et al. 1999; Steidel et al. 2000; Francis et al. 2001; Matsuda et al. 2004; Palunas et al. 2004; Dey et al. 2005; Villar-Martin et al. 2005). At least three mechanisms have been suggested as energy sources for Ly $\alpha$ blobs. These are: $i$ ) hidden QSOs (Haiman \& Rees 2001; Weidinger et al. 2004, 2005); ii) star formation and superwinds from (possibly obscured) starburst galaxies (Taniguchi et al. 2001; Ohyama et al. 2003; Mori et al. 2004; Wilman et al. 2005); and iii) socalled cold accretion (Haiman et al. 2000; Fardal et al. 2001; Keres et al. 2004; Maller \& Bullock 2004; Birnboim \& Dekel 2003; Sommer-Larsen 2005; Dijkstra et al. 2006a,b; Dekel \& Birnboim 2006). Cooling flows are phenomena observed in galaxy clusters for more than a decade (Fabian 1994). These are explained by gas which is cooling much faster than the Hubble time through X-ray emission in the centres of the clusters. However, cooling emission from a galaxy, or a group sized halo can be dominated by Ly $\alpha$ emission (e.g. Haiman et al. 2000; Dijkstra et al. 2006a,b). In this letter we present the discovery of a Ly $\alpha$ blob at redshift $z \approx 3.16$ located in the GOODS South field, which we argue is the first piece of evidence for cold gas accretion onto a dark matter halo.

Throughout this paper, we assume a cosmology with $H_{0}=$ $72 \mathrm{~km} \mathrm{~s}^{-1} \mathrm{Mpc}^{-1}, \Omega_{\mathrm{m}}=0.3$ and $\Omega_{\Lambda}=0.7$. All magnitudes are in the $\mathrm{AB}$ system.

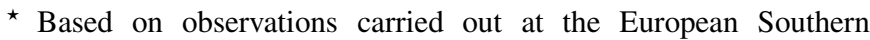
Observatory (ESO) under prog. ID No. 70.A-0447 and 274.A-5029.

\section{Observations and data reduction}

A $400 \times 400 \operatorname{arcsec}^{2}$ section, centred on RA $=03^{\mathrm{h}} 32^{\mathrm{m}} 21.8^{\mathrm{s}}$, Dec $=-27^{\circ} 45^{\prime} 52^{\prime \prime}(\mathrm{J} 2000)$, of the GOODS South field was observed with FORS1 on the VLT $8.2 \mathrm{~m}$ telescope Antu during two visitor mode nights on December 1-3, 2002. A total of 16 dithered exposures were obtained over the two nights for a combined exposure time of $30 \mathrm{ks}$, all with the narrow band filter OIII/3000+51 and using the standard resolution collimator $\left(0.2 \times 0.2 \operatorname{arcsec}^{2}\right.$ pixels $)$. For this setup the central wavelength of the filter is $5055 \AA$ with a $F W H M$ of $59 \AA$, corresponding to the redshift range $z=3.126-3.174$ for $\operatorname{Ly} \alpha$.

The observing conditions were unstable during the two nights with the seeing $F W H M$ varying between $0 . ' 66$ and 1'.25 on the first night and 1". 4 and 3". 3 on the second night. The images were reduced (de-biased, and corrected for CCD pixel-topixel variations using twilight flats) using standard techniques. The individual reduced images were combined using a modified version of our code that optimizes the Signal-to-Noise $(\mathrm{S} / \mathrm{N})$ ratio for faint, sky-dominated sources (see Møller \& Warren 1993, for details on this code). The modification of the code was necessitated by the highly variable seeing. The sky background was assumed to be constant. The $F W H M$ of the PSF of the final combined narrow-band image is 0 ' $^{\prime} 8$.

For object detection, we used the software package SExtractor (Bertin \& Arnouts 1996). A full description of our selection of $\operatorname{Ly} \alpha$ emitters in the GOODS field will be given in a subsequent paper. In this Letter we discuss the nature of an extended, low surface brightness blob with a centroid (of the Ly $\alpha$ emission) of RA $=03^{\mathrm{h}} 32^{\mathrm{m}} 14.6^{\mathrm{s}}$ and Dec $=-27^{\circ} 43^{\prime} 02^{\prime \prime} .4$ (J2000) detected in the combined narrow-band image.

Follow-up MOS spectroscopy was obtained in service mode using FORS1/VLT UT2 over the time period 
Table 1. Specifications of deep, multi-wavelength data available in the GOODS South field and the narrow-band image. The last column gives the $3 \sigma$ limit as detected in a $2^{\prime \prime}$ radius aperture and the narrow-band value gives the blob flux in this aperture.

\begin{tabular}{lccc}
\hline \hline Filter/Channel & $\lambda_{\mathrm{c}}$ & $\begin{array}{c}\text { Filter } \\
F W H M\end{array}$ & $\begin{array}{c}3 \sigma \text { limit }\left(2^{\prime \prime} \text { aperture }\right) \\
\left(\mathrm{erg} \mathrm{cm}^{-2} \mathrm{~s}^{-1} \mathrm{~Hz}^{-1}\right)\end{array}$ \\
\hline X-rays $($ Chandra $)$ & $4.15 \mathrm{keV}$ & $3.85 \mathrm{keV}$ & $9.90 \times 10^{-34}$ \\
$U($ ESO $2.2-\mathrm{m})$ & $3630 \AA$ & $760 \AA$ & $8.62 \times 10^{-31}$ \\
$B(H S T)$ & $4297 \AA$ & $1038 \AA$ & $9.25 \times 10^{-30}$ \\
Narrow $(V L T)$ & $5055 \AA$ & $60 \AA$ & $6.68 \times 10^{-30}$ \\
$V(H S T)$ & $5907 \AA$ & $2342 \AA$ & $4.66 \times 10^{-30}$ \\
$i(H S T)$ & $7764 \AA$ & $1528 \AA$ & $1.50 \times 10^{-29}$ \\
$z(H S T)$ & $9445 \AA$ & $1230 \AA$ & $3.00 \times 10^{-29}$ \\
$J($ VLT $)$ & $1.25 \mu \mathrm{m}$ & $0.6 \mu \mathrm{m}$ & $5.31 \times 10^{-30}$ \\
$H($ VLT $)$ & $1.65 \mu \mathrm{m}$ & $0.6 \mu \mathrm{m}$ & $1.86 \times 10^{-29}$ \\
$K \mathrm{~s}($ VLT $)$ & $2.16 \mu \mathrm{m}$ & $0.6 \mu \mathrm{m}$ & $1.56 \times 10^{-29}$ \\
Ch1 $($ Spitzer/IRAC $)$ & $3.58 \mu \mathrm{m}$ & $0.75 \mu \mathrm{m}$ & $2.51 \times 10^{-31}$ \\
Ch2 (Spitzer/IRAC) $)$ & $4.50 \mu \mathrm{m}$ & $1.02 \mu \mathrm{m}$ & $6.43 \times 10^{-32}$ \\
Ch3 $($ Spitzer/IRAC $)$ & $5.80 \mu \mathrm{m}$ & $1.43 \mu \mathrm{m}$ & $5.01 \times 10^{-29}$ \\
Ch4 (Spitzer/IRAC $)$ & $8.00 \mu \mathrm{m}$ & $2.91 \mu \mathrm{m}$ & $4.65 \times 10^{-30}$ \\
\hline
\end{tabular}

December 2004-February 2005. The total observing time was 6 hours. We used a 1". 4 slitlet and grism $600 \mathrm{~V}$ resulting in a wavelength range of $4650 \AA$ to $7100 \AA$ and a spectral resolution $F W H M$ of approximately 700 . The seeing varied between 0.77 and 1 .'2 during the spectroscopic observations.

The GOODS archival data used here and their detection limits are listed in Table 1.

\section{Results}

The spectrum of the part of the Ly $\alpha$ blob covered by the slitlet can be seen in the left-most panel of Fig. 1. The line has the asymmetric profile expected for a high redshift Ly $\alpha$ emitter. We detect no other emission lines in the spectrum. The most likely interloper is [OII] at redshift 0.36 , but no emission is observed in the spectrum where e.g. $\mathrm{H} \beta$ or $[\mathrm{OIII}]$ are expected at this redshift, see Fig. 1. This leads us to the conclusion that we are observing a Ly $\alpha$-emitting object at $z=3.157$. The observed $F W H M$ velocity width of the emission line is $505 \mathrm{~km} \mathrm{~s}^{-1}$. The instrument $F W H M$ of the set-up is $290 \mathrm{~km} \mathrm{~s}^{-1}$, hence the Ly $\alpha$ intrinsic velocity width is marginally resolved. The intrinsic width is less than $500 \mathrm{~km} \mathrm{~s}^{-1}$. This is of the order or smaller than for other published blobs, with velocity widths of 500-2000 $\mathrm{km} \mathrm{s}^{-1}$ (Keel et al. 1999; Steidel et al. 2000; Francis et al. 2001; Ohyama et al. 2003; Bower et al. 2004; Dey et al. 2005).

A contour-plot of the blob superimposed on the HST/ACS $V$-band image is shown in the middle panel of Fig. 1 and a plot of the surface brightness of the blob is seen in the right panel of the same figure. The full set of thumb-nail images of the blob in all 14 bands can be found in Fig. 2. No obvious continuum counterpart is detected in any band. The radial size is at least $30 \mathrm{kpc}$ (60 kpc diameter) with fainter emission extending out to $40 \mathrm{kpc}$ radius. This can be seen as the extension to the SW in the contour-plot in Fig. 1. The significance of the lowest contour levels is of the order of $2 \sigma$ per pixel. The total Ly $\alpha$ luminosity, in a $30 \mathrm{kpc}$ radius aperture, is $L_{\mathrm{Ly} \alpha}=(1.09 \pm 0.07) \times 10^{43} \mathrm{erg} \mathrm{s}^{-1}$. This coincides, after correction for the smaller area sampled in the spectrum, to the Ly $\alpha$ flux detected in the spectrum within errors. A conservative lower limit to the restframe equivalent width $(E W)$ of the emission line can be calculated from upper limits on the broad-band fluxes in the HST $B$ and $V$ filters in the
Table 2. Photometric redshifts of objects surrounding the blob. Numbering refers to those given in Fig. 1. Errors given are $1 \sigma$.

\begin{tabular}{lccccc}
\hline \hline Obj \# & $\begin{array}{c}\text { Dist. from blob } \\
(\operatorname{arcsec})\end{array}$ & $z_{\text {phot }}$ & $\chi^{2} /$ d.o.f. & Type & $A_{\mathrm{V}}$ rest \\
\hline 1 & 4.6 & $1.1_{-0.30}^{+0.40}$ & 1.3 & Burst & 0.20 \\
2 & 4.6 & $1.1_{-0.41}^{+0.34}$ & 8.6 & Burst & 1.20 \\
3 & 6.8 & $2.9_{-0.59}^{+1.41}$ & 4.8 & Spiral & 1.20 \\
4 & 8.4 & $0.6_{-0.63}^{+1.97}$ & 8.1 & Burst & 0.40 \\
5 & 8.7 & $0.9_{-0.89}^{+2.46}$ & 2.0 & Burst & 0.20 \\
6 & 3.0 & $4.5_{-1.54}^{+4.29}$ & 0.9 & Spiral & 1.20 \\
7 & 6.3 & $1.1_{-0.81}^{+1.24}$ & 1.9 & Burst & 1.20 \\
8 & 4.5 & $3.5_{-3.48}^{+1.27}$ & 0.6 & Spiral & 0.00 \\
\hline
\end{tabular}

same aperture. This limit is $E W \gtrsim 220 \AA$ in the restframe. This is in the range of previously published Ly $\alpha$ blobs, that have a Ly $\alpha$ flux to $B$-band flux density range between $50-1500 \AA$ in the restframe (but typically these values are derived measuring the continuum flux in a smaller aperture than the emission line flux).

There are seven objects detected in a wide range $(\geq 8)$ of energy bands, within a $10^{\prime \prime}$ radius surrounding the blob. 8 other objects are detected within the $V$-band and one further detected in the Spitzer/IRAC bands. The photometric redshift of these objects was calculated using the public Hyper ${ }^{1}$ code by Bolzonella et al. (2000). The resulting photometric redshifts for the eight objects with most data points $(\geq 8)$ can be found in Table 2 . The other eight objects detected in the $V$-band have only a few detections across the spectrum and hence their photometric redshifts are unreliable. The redshift of object \#3 is similar to the blob redshift and indicates that this galaxy may be near to the blob. Object \#6 is an intriguing object, undetected in the deep optical and near-IR imaging but bright in the Spitzer/IRAC bands. Its photometric redshift is consistent with the redshift of the blob, but with a large uncertainty. The object is also detected in the Spitzer/MIPS $24 \mu \mathrm{m}$ band. Based on the Spitzer magnitudes, and on the diagnostic colour-colour diagram of Ivison et al. (2004), object \#6 is best fit by a star-burst at high redshift ( $z \sim 5.5$, consistent with the photometric redshift estimate), hence unrelated to the blob.

\section{Discussion}

We first consider that the $\operatorname{Ly} \alpha$ emission of the blob may be due to recombination of gas, photo-ionized by an AGN or a starburst galaxy. If the blob is a "passive" gas cloud illuminated and photo-ionized by a nearby AGN, then, following Cantalupo et al. (2005), one can show that for an AGN with luminosity $L_{v}=L_{\mathrm{LL}}\left(v / v_{\mathrm{LL}}\right)^{-\alpha}$, and in order to result in a peak blob Ly $\alpha$ surface brightness of $\Sigma_{\mathrm{Ly} \alpha}$, the AGN has to be located at a distance of no more than

$270 \mathrm{kpc}\left(\frac{\Sigma_{\mathrm{Ly} \alpha}}{10^{-3} \mathrm{erg} \mathrm{s}^{-1} \mathrm{~cm}^{-2}}\right)^{-1} \sqrt{\frac{L_{\mathrm{LL}}}{10^{30} \mathrm{erg} \mathrm{s}^{-1} \mathrm{~Hz}^{-1}}} \sqrt{\frac{0.7}{\alpha}}$

where equality applies to the case where the blob gas is optically thick at the Lyman limit. No AGN has been detected in the deep Chandra image available within this distance. Worsley et al. (2005) argue that a significant fraction of the unresolved X-ray background in hard bands consists of highly obscured AGN. However, Worsley et al. (2005) also predict that the AGN responsible for this background are situated at $z \sim 0.5-1.0$.

\footnotetext{
${ }^{1}$ http://webast .ast.obs-mip.fr/hyperz/
} 

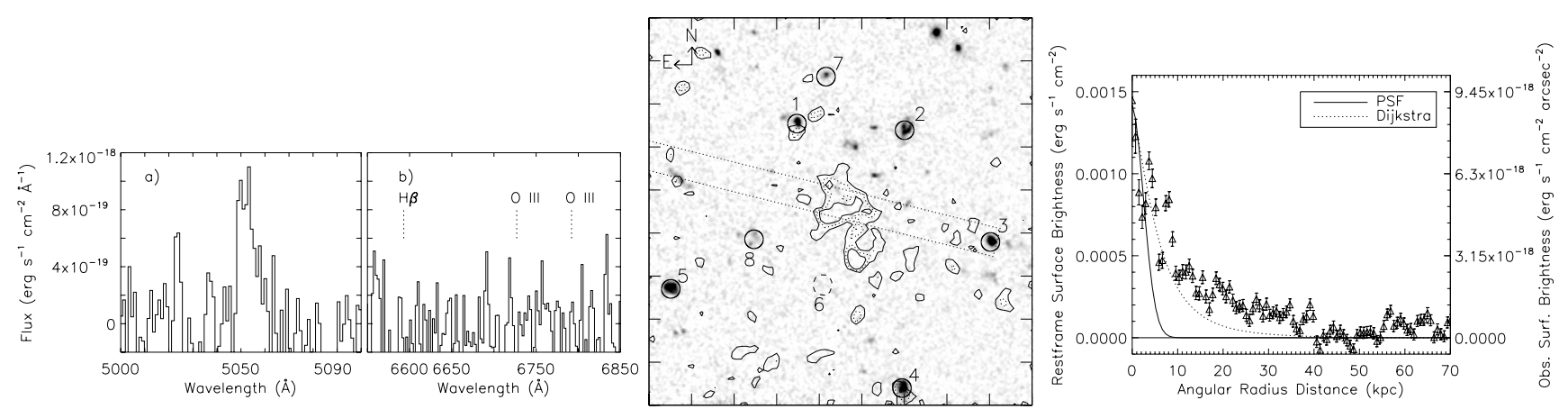

Fig. 1. Left: a) flux calibrated spectrum of the blob emission line. The line has the characteristic blue side absorption, indicating high redshift. b) The part of the spectrum (binned with a binsize equal to half the resolution (1.1 $\AA$ )) where $\mathrm{H} \beta$ and [OIII] should have been observed if the emission line was [OII] at a redshift of $z \approx 0.36$. These lines are not observed and therefore we conclude the observed line is due to Ly $\alpha$ at $z=3.16$. Middle: contour-plot of narrow-band emission from the Ly $\alpha$ blob overlaid the HST $V$-band image. The narrow-band image has been continuum subtracted by subtracting the re-binned, smoothed and scaled HST/V-band image. Contour levels are $2 \times 10^{-4}, 4 \times 10^{-4}$ and $6 \times 10^{-4} \mathrm{erg} \mathrm{s}^{-1} \mathrm{~cm}^{-2}$ in restframe flux (corresponding to $1.2 \times 10^{-18}, 2.5 \times 10^{-18}$ and $3.7 \times 10^{-18}$ in observed flux). The image is $18^{\prime \prime} \times 18^{\prime \prime}\left(18^{\prime \prime}\right.$ corresponds to a physical size of $\sim 133 \mathrm{kpc}$ ). Numbers refer to those used in Sect. 3. The dotted lines indicate the slitlet position for our follow-up spectroscopy. Right: plot of surface brightness as function of radius. The flux is the sky subtracted narrow-band flux. The PSF of the image is illustrated by the solid line, and the dotted line is the best fit model of Dijkstra et al. (2006). The deficit at $\sim 45 \mathrm{kpc}$ is due to the asymmetric appearance of the blob.

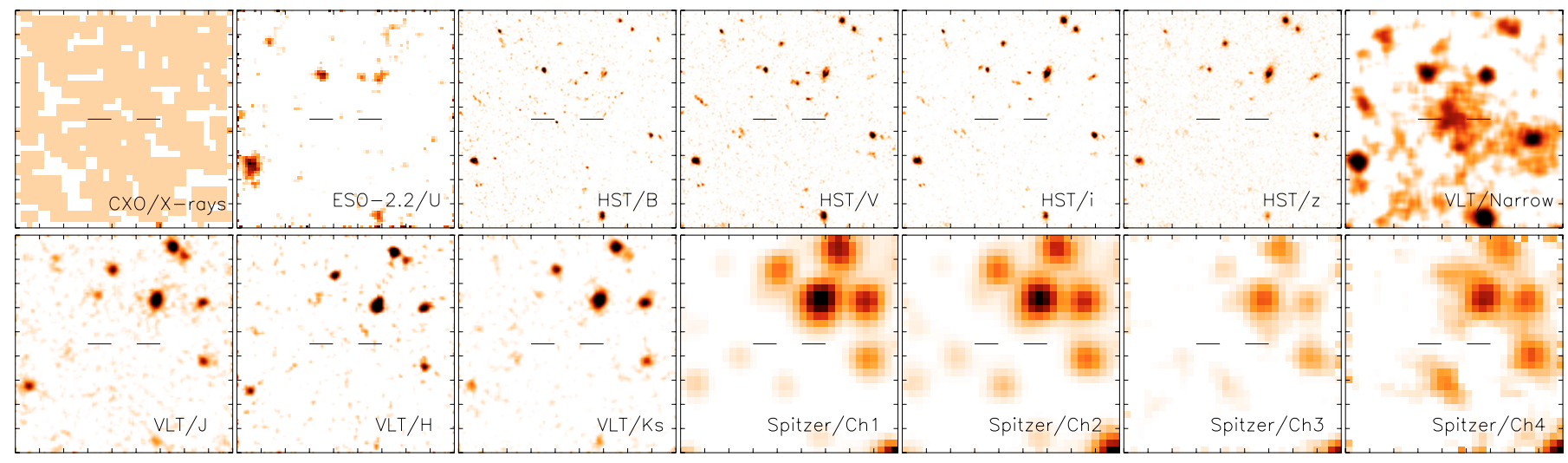

Fig. 2. Thumbnail images of all available multi-wavelength data in the GOODS South field, centred on the Ly $\alpha$ blob. All images are $18^{\prime \prime} \times 18^{\prime \prime}$.

Furthermore, Silverman et al. (2005) present a luminosity function for AGN at higher redshift. To the detection limit of the $\operatorname{CDFS}\left(L_{X}(z=3.15) \approx 1.9 \times 10^{43} \mathrm{erg} \mathrm{s}^{-1}\right)$ and with our search volume $(3 \times 3 \mathrm{Mpc} \times \Delta z=0.05)$ we expect to detect only 0.06 AGN in our entire search volume. We also consider the possibility that galaxy \#3 can photo-ionise the blob. However, if we assume a power law for the spectrum and extrapolating from the HST/B and HST/V detections we find that the UV luminosity of galaxy \#3 is not sufficient to photo-ionise the blob, unless highly collimated towards the blob. We have no reason to believe that this is the case.

The second possibility is that the blob $\operatorname{Ly} \alpha$ emission is somehow related to starburst driven, superwind outflows. A starburst would be expected to be located within the blob to create such a Ly $\alpha$ halo and no central continuum source has been detected. Even though a very massive starburst can be made invisible in the UV/optical range by dust obscuration, it should be visible in the IR, i.e. the Spitzer/IRAC bands.

The third option is that the Ly $\alpha$ emission is due to cold accretion of predominantly neutral, filamentary gas onto a massive dark matter halo. For cold accretion, the bulk of the Ly $\alpha$ emission is produced by collisional excitation, rather than recombination. Recently, Dijkstra et al. (2006a,b) presented a theoretical model for Ly $\alpha$ cooling flows, along with predictions of the emission line profile and the shape of the surface brightness function. The $\mathrm{S} / \mathrm{N}$ of our spectrum is not high enough to allow a comparison of emission line profiles. However, the surface brightness profile matches well the predictions for a centrally illuminated, collapsing cloud of Dijkstra et al. (2006a), see Fig. 1. Further tests are needed to determine how well their model fits. To test whether this blob can be filamentary gas accreting "cold" onto a companion galaxy, we also conducted the following experiment: we calculated the Ly $\alpha$ surface brightness in a $100 \times 100 \mathrm{kpc}$ (projected) region for a proto-galaxy of "cooling" radiation only (so all contributions from regions with young stars were removed, as well as all emission, in general, from gas closer than $10 \mathrm{kpc}$ to any star-forming region). The calculation was based on a cosmological simulation of the formation and evolution of an M31-like disk galaxy (Sommer-Larsen 2005; Portinari \& Sommer-Larsen 2005).

The results at $z \sim 3$ are presented in Sommer-Larsen (2005), and get to a surface brightness about an order of magnitude lower than the observed level. This is interesting, and may point to a cold accretion origin of the blob Ly $\alpha$ emission on a larger scale, such as filamentary gas accretion onto a galaxy-group sized halo. Another possibility is that the periods with high surface brightness are shorter than 2.5 Myr (the resolution of the simulation). Given that in a search volume of about 40000 co-moving $\mathrm{Mpc}^{3}$, 
only one such blob has been detected, it is actually comforting, that we could not reproduce the blob characteristics, by cold accretion onto this, randomly selected, M31-like galaxy. This has to be a rare phenomenon.

A test for the cold accretion model would be to observe the Balmer lines. For collisionally excited hydrogen, neglecting extinction effects, the flux in $\mathrm{H} \alpha$ should only be about 3.5 percent of the Ly $\alpha$ flux, whereas for recombining, photo-ionized gas this ratio is $\sim 11.5 \%$ (Brocklehurst 1971). Hence, the relative $\mathrm{H} \alpha$ luminosity is expected to be significantly larger in the latter case. The situation is similar for $\mathrm{H} \beta$, and whereas the $\mathrm{H} \alpha$ line will be very difficult to detect from the ground, $\mathrm{H} \beta$ should be observable.

\section{Conclusion}

We have here reported the results of an extensive multiwavelength investigation of a redshift $z=3.16$ Ly $\alpha$ emitting blob discovered in the GOODS South field. The blob has a diameter larger than $60 \mathrm{kpc}$ diameter and a total luminosity of $L_{\mathrm{Ly} \alpha} \sim 10^{43} \mathrm{erg} \mathrm{s}^{-1}$. Deep HST imaging show no obvious optical counterpart, and the lack of X-ray or IR emission suggest there are no AGN or dusty starburst components associated with at least the centroid of the blob. Two galaxies within a $10^{\prime \prime}$ radius have photometric redshifts consistent with the redshift of the blob, but follow-up spectroscopy is needed to establish if there is a connection. We have run simulations of Ly $\alpha$ surface brightness arising from cold accretion and found that such extended $\operatorname{Ly} \alpha$ emission may be explained by accretion along a filament onto a galaxy group sized dark matter halo. Another possibility is that such emission in very short lived, i.e. significantly shorter than the $2.5 \mathrm{Myr}$ resolution of our simulation. We argue that other previously suggested origins of Ly $\alpha$ blobs (hidden AGN and "super-winds") can be ruled out in this case due to the lack of detected continuum counter-parts. Hence, though our cold accretion simulation cannot perfectly match our data, it is the only explanation that is plausible. Our results combined with the fact that previously studied blobs appear to be caused by superwinds and/or AGN in turn implies that the energy sources for blob Ly $\alpha$ emission are diverse.

Acknowledgements. K.N. gratefully acknowledges support from IDA Instrumentcentre for Danish Astrophysics. The numerical simulations used in this paper were performed on the facilities provided by Danish Center for
Scientific Computing (DCSC). The Dark Cosmology Centre is funded by the DNRF. The authors thank the DDT panel and the ESO Director General for granting time for follow-up spectroscopy.

\section{References}

Bertin, E., \& Arnouts, S. 1996, A\&AS, 117, 393

Birnboim, Y., \& Dekel, A. 2003, MNRAS, 345, 349

Bolzonella, M., Miralles, J.-M., \& Pelló, R. 2000, A\&A, 363, 476

Bower, R. G., Morris, S. L., Bacon, R., et al. 2004, MNRAS, 351, 63

Brocklehurst, M. 1971, MNRAS, 153, 471

Cantalupo, S., Porciani, C., Lilly, S. J., \& Miniati, F. 2005, ApJ, 628, 61

Dekel, A., \& Birnboim, Y. 2006, MNRAS, in press [arXiv: astro-ph/0412300]

Dey, A., Bian, C., Soifer, B. T., et al. 2005, ApJ, 629, 654

Dijkstra, M., Haiman, Z., \& Spaans, M. 2006a, ApJ, submitted [arXiv:astro-ph/0510407]

Dijkstra, M., Haiman, Z., \& Spaans, M. 2006b, ApJ, submitted [arXiv: astro-ph/0510409]

Fabian, A. C. 1994, ARA\&A, 32, 277

Fardal, M. A., Katz, N., Gardner, J. P., et al. 2001, ApJ, 562, 605

Francis, P. J., Williger, G. M., Collins, N. R., et al. 2001, ApJ, 554, 1001

Haiman, Z., Spaans, M., \& Quataert, E. 2000, ApJ, 537, L5

Haiman, Z., \& Rees, M. J. 2001, ApJ 556, 87

Fynbo, J. P. U., Møller, P., \& Warren, S. J. 1999, MNRAS, 305, 849

Ivison, R. J., Greve, T. R., Serjeant, S., et al. 2004, ApJS, 154, 124

Keel, W. C., Cohen, S. H., Windhorst, R. A., \& Waddington, I. 1999, AJ, 118, 2547

Keres, D., Katz, N., Weinberg, D. H., \& Dave, R. 2005, MNRAS, 363, 2

Maller, A. H., \& Bullock, J. S. 2004, MNRAS, 355, 694

Matsuda, Y., Yamada, T., Hayashino, T., et al. 2004, AJ, 128, 569

Møller, P., \& Warren, S. J. 1993, A\&A, 270, 43

Mori, M., Umemura, M., \& Ferrara, A. 2004, ApJ, 613, L97

Ohyama, Y., Taniguchi, Y., Kawabata, K. S., et al. 2003, ApJ, 591, L9

Palunas, P., Teplitz, H. I., Francis, P. J., Williger, G. M., \& Woodgate, B. E. 2004, ApJ, 602, 545

Portinari, L., \& Sommer-Larsen, J. 2005, MNRAS, to be submitted

Silverman, J., Green, P., Barkhouse, W., et al. 2005, Proc. The X-ray Universe 2005, San Lorenzo de El Escorial, Spain, September 2005, to appear [arXiv: astro-ph/0511552]

Sommer-Larsen, J. 2005, in the proceedings of the Island Universes: Structure and Evolution of Disk Galaxies, conference held in Terschelling, Netherlands, July 2005, ed. R. de Jong (Dordrecht: Springer) [arXiv: astro-ph/0512485]

Steidel, C. C., Adelberger, K., Shapley, A. E., et al. 2000, ApJ, 532, 170

Taniguchi, Y., Shioya, Y., \& Kakazu, Y. 2001, ApJ, 562, L15

Villar-Martin, Sanchez, S. F., \& De Breuck, C. 2005, MNRAS, 359, L5

Weidinger, M., Møller, P., \& Fynbo, J. P. U. 2004, Nature, 430, 999

Weidinger, M., Møller, P., Fynbo, J. P. U., \& Thomsen, B. 2005, A\&A, 436, 825

Wilman, R. J., Gerssen, J., Bower, R. G., et al. 2005, Nature, 436, 14

Worsley, M. A., Fabian, A. C., Bauer, F. E., et al. 2005, MNRAS, 357, 1281 